\title{
Editorial: Chris Leighton Introduces Physical Review Materials
}

Materials research is arguably one of the most active and dynamic areas in contemporary science. While fundamentally interdisciplinary, research on materials is also inextricably linked to physics, and it is thus fitting that materials feature prominently in several APS journals, including Physical Review Letters (PRL), Physical Review X (PRX), Physical Review Applied (PR Applied), Physical Review B (PRB), and Physical Review $E$ (PRE). The Physical Review has never featured, however, a journal with an explicit focus on materials. This changed on April $4^{\text {th }}$ of this year, when Physical Review Materials (PR Materials), the newest member of the Physical Review family, was launched. Born from a deliberate process of reflection, analysis, and information gathering, with considerable input from the APS community, PR Materials aims to fully embrace research in materials, best serving the broad international community of researchers active in this area. This community is increasingly interdisciplinary, encompassing physicists, materials scientists, chemists, and engineers, with a shared interest in the prediction, synthesis, processing, structure, properties, and performance of a staggering variety of materials. PR Materials will reflect each of these areas, publishing high quality original research in theoretical and experimental areas (as both Regular Articles and Rapid Communications), in addition to Reviews of emerging fields.

I am proud and excited to be the inaugural Lead Editor of PR Materials. This is a role I will work in mostly remotely, as I continue my faculty position in the Department of Chemical Engineering and Materials Science at the University of Minnesota. My initial goals with the journal are many-fold, and will no doubt evolve as the journal develops in response to our community of authors. First, we of course plan to continue the tradition of excellence APS maintains in the review and publication processes at each of its journals. The specific goal with PR Materials is to create a sibling to the Physical Review A-E journals, maintaining their high editorial standards and practices, but with a specific focus on materials. While some overlap with journals such as PRB, PRE, and PR Applied is thus inevitable, PR Materials will embrace a much broader scope of research in materials, thus appealing to authors and communities that may not currently publish regularly in the Physical Review. We believe that this will not only better serve the international materials research community, but will also strengthen the APS publishing effort as a whole. Alongside this, we will also of course emphasize a fair review process that is as expeditious as possible, emphasizing high quality, and relevance to materials research. We hope that this will establish in the Physical Review family a leading venue for materials research, with deep scientific impact.

To this end, the APS and PR Materials staff have been hard at work for the last two months handling a volume of submissions that already indicates significant interest from the community. Managing Editor Athanasios Chantis, Editor Mu Wang, along with myself as Lead Editor, provide a mix of expert professional editors and research scientists active in the community, which we plan to maintain as the journal expands. An Editorial Board of leading scientists from around the world provides the other key element in our operation. This board currently numbers 22 outstanding researchers from the US, Europe, Asia, and Australia, representing universities, national laboratories, user facilities, and research institutes. These Editorial Board Members, who will increase in number as the journal expands, span a diverse range of backgrounds and interests, and will provide invaluable direction and input to the PR Materials editors, for which we are grateful. The efforts of our entire team - editorial staff, journal operations staff, and board members - are reflected in this first issue of PR Materials papers, which I hope you will read with interest. I believe this is an outstanding first batch of publications, beautifully illustrating the high quality and broad scope of the research we plan to publish.

PR Materials owes its existence to the large number of people at APS who have played substantial roles in its inception, and I would like to take this opportunity to thank each of them. Particular acknowledgment is appropriate for APS Editor in Chief Pierre Meystre, APS Publisher Matthew Salter, Editorial Director Daniel Kulp, PR Materials Managing Editor Athanasios Chantis, Assistant Editorial Director Luigi Longobardi, 
Chief Information Officer Mark Doyle, Director of Journal Operations Christine Giaccone, and the PRB team headed by Laurens Molenkamp and Anthony Begley, including Hari Dahal, Brad Rubin, and Sarma Kancharla. Many additional editorial staff, journal operations staff, and APS volunteers played important roles, and are also graciously acknowledged.

Thank you in advance for your support, manuscript submissions, and reviewing efforts on behalf of PR Materials.

Chris Leighton Editor

Published 19 June 2017

DOI: 10.1103/PhysRevMaterials.1.010002 